\title{
Thin film surface processing by ultrashort laser pulses (USLP)
}

\author{
D. Scorticati ${ }^{1 \mathrm{a}}$, J.Z.P. Skolski ${ }^{\mathrm{a}, \mathrm{b}}$, G.R.B.E. Römer ${ }^{\mathrm{a}}$, A.J. Huis in 't Veld ${ }^{\mathrm{a}, \mathrm{d}}$, M. Workum ${ }^{\mathrm{c}}$, M. \\ Theelen ${ }^{\mathrm{c}, \mathrm{d}}$, M. Zeman ${ }^{\mathrm{c}}$ \\ ${ }^{a}$ University of Twente, Faculty of Engineering Technology, 7500AE Enschede, NL; \\ ${ }^{b}$ Materials innovation institute (M2i), 7522NB Enschede, NL; \\ ${ }^{\mathrm{c}}$ Delft University of Technology, Photovoltaic Materials and Devices, Delft, NL; \\ ${ }^{\mathrm{d}}$ TNO Technical Sciences; Mechatronics, Mechanics and Materials, 5600AE Eindhoven, NL
}

\begin{abstract}
In this work, we studied the feasibility of surface texturing of thin molybdenum layers on a borosilicate glass substrate with Ultra-Short Laser Pulses (USLP). Large areas of regular diffraction gratings were produced consisting of Laserinduced periodic surface structures (LIPSS).

A short pulsed laser source $\left(230 f_{s}-10 \mathrm{ps}\right)$ was applied using a focused Gaussian beam profile (15-30 $\left.\mu m\right)$. Laser parameters such as fluence, overlap (OL) and Overscans (OS), repetition frequency (100-200 kHz), wavelength (1030 $\mathrm{nm}, 515 \mathrm{~nm}$ and $343 \mathrm{~nm}$ ) and polarization were varied to study the effect on periodicity, height and especially regularity of LIPSS obtained in layers of different thicknesses $(150-400 \mathrm{~nm})$. The aim was to produce these structures without cracking the metal layer and with as little ablation as possible.

It was found that USLP are suitable to reach high power densities at the surface of the thin layers, avoiding mechanical stresses, cracking and delamination.

A possible photovoltaic (PV) application could be found in texturing of thin film cells to enhance light trapping mechanisms.
\end{abstract}

Keywords: Keywords: Ultra Short Laser Pulses, surface processing, molybdenum, thin film, ripples, LIPSS, $p s$ laser

\section{INTRODUCTION}

Laser-induced periodic surface structures, also referred to as ripples, are wavy structures observed on the surface of many materials after laser irradiation. Although LIPSS have been studied since 1965 [2], their complete explanation is still debated. The regularity, shape and dimensions of LIPSS depend on the laser irradiation conditions as well as material properties. Periodicity, amplitude and orientation of LIPSS depend on the laser's wavelength, fluence and polarization $[15,19]$. Under most processing conditions, LIPSS show bifurcations or forking (when a single ripple split in two of half periodicity) and their length is smaller than the spot size; but interestingly, by choosing proper process parameters, the length of LIPSS can be extended without interruptions to areas larger than the spot size. Highly periodical-i.e. very regular- LIPSS, longer than the laser spot size, have been shown in "scanned laser lines" on different bulk materials [4]. A practical application of these highly regular wavy structures, without bifurcations, could be found in diffraction nano-gratings.

Nano-gratings of different periodicities using this technique have been obtained on bulk materials [3], while the feasibility of this process on thin deposited metal films ( $\leq 400 \mathrm{~nm}$ in our work) has not been yet studied extensively.

Besides finding the proper processing conditions for obtaining a uniform area of LIPSS on a given material, the processing of thin metallic films adds another complexity, because thermo-mechanical effects during processing may cause cracking, delamination and excessive ablation of the thin layer. Fortunately, the so-called Brittle to Ductile Transition (BDT) [20] allows thin metallic layers to undergo thermal stresses during laser processing without cracking.

\footnotetext{
1 *D.Scorticati@utwente.nl; phone +31 53 489-3532; www.utwente.nl
}

Photonics for Solar Energy Systems IV, edited by Ralf Wehrspohn, Andreas Gombert

Proc. of SPIE Vol. 8438, 84380T - (C) 2012 SPIE · CCC code: 0277-786X/12/\$18 · doi: 10.1117/12.922270 
The aim of the present research was to show the possibility to create highly regular LIPSS using ultra-short laser pulses on the top surface of molybdenum (Mo) layers of $400 \mathrm{~nm}$ thickness on glass, without cracking the layer or having excessive ablation, which would expose the supporting glass substrate (see section 3 ).

Moreover, we presented the feasibility of those structures on thin Mo films using $p s$ lasers instead of $f_{s}$ lasers, which makes the technology more attractive for industrial applications. The periodicity of the experimentally obtained LIPSS was compared to an analytical model of Sipe et al. [13], describing the absorbed laser energy below bulk material's rough surface.

\section{EXPERIMENTAL SETUP}

\subsection{Laser setup}

Two different laser sources were employed to study the effect of fluence, pulse duration and wavelength on LIPSS formation in the Mo layer:

- The Pharos source from Light Conversion Ltd, which is a $\mathrm{Yb}: \mathrm{KGW}$ laser source with tunable pulse length (230 $f s-10 \mathrm{ps}), 10 \mathrm{~W}$ maximum output, central wavelength of $1030 \mathrm{~nm}$, maximum repetition frequency $200 \mathrm{kHz}$ and a beam quality $\mathrm{M}^{2}<1.2$.

- The TruMicro 5050 from Trumpf, which is a Yb:YAG laser source, with fixed pulse length of $6.7 \mathrm{ps}, 50 \mathrm{~W}$ maximum output, central wavelength of $1030 \mathrm{~nm}$ (IR) maximum repetition frequency of $400 \mathrm{kHz}$ and $\mathrm{M}^{2}<1.3$.

Both laser sources showed a Gaussian power density distribution and were equipped with pulse-pickers, allowing the user to change the repetition frequency without affecting the energy per pulse. To study the effect of the laser wavelength, a Second Harmonic Generation (SHG) or a Third Harmonic Generation (THG) unit was employed to convert the central wavelength to $515 \mathrm{~nm}$ (green) and $343 \mathrm{~nm}(U V)$.

The diameter $d$ of the focused laser beam on the surface was determined using the $\mathrm{D}^{2}$ method [4] for each wavelength used. $\mathrm{d}$ was found to range from 15 to $30 \mu \mathrm{m}$, depending on the wavelength and setup used. Fluence was then calculated for single pulses and the OL was defined as:

$$
\mathrm{OL}=1-v /\left(d \cdot f_{\mathrm{p}}\right),
$$

where $v$ denotes the velocity of the focal spot, and $f_{\mathrm{p}}$ the applied pulse frequency.

In both systems, galvano-scanners (IntelliScan14 of Scanlab) were used to scan the focal spot over the surface of the samples with telecentric F-theta lenses (Ronar of Linos) to focus the laser beam on the samples. The focal length of theta lenses used with the TruMicro laser for IR, green and UV were respectively $80 \mathrm{~mm}, 100 \mathrm{~mm}$ and $100 \mathrm{~mm}$, while with the Pharos source, only IR was exploited and the focal length for the lens used was $100 \mathrm{~mm}$.

\subsection{Analysis equipment}

Morphological inspection of the laser-treated areas was performed by optical microscopy, Scanning Electron Microscopy SEM, JCM-5000 NeoScope), Atomic Force Microscopy (AFM, Nanosurf easyscan 2), Confocal Laser Scanning Microscopy (CLSM, Keyence VK-9700). A spectrophotometer (PerkinElmer Lambda 950 with ARTA accessory) was used to analyze the angular intensity distribution (AID) of the refracted light at different angles and wavelengths of the obtained nano-gratings [5].

\subsection{Samples}

Mo layers of different thicknesses were deposited on glass substrates. For initial tests, samples of $150 \mathrm{~nm}$ thickness deposited by Physical Vapor Deposition (PVD) on borosilicate glass were used. For structuring areas with LIPSS, we used Mo layers deposited by sputtering on soda lime glass by third party. The latter combination of thin film Mo on soda lime glass is regularly used for the production of $\mathrm{Cu}(\mathrm{In}, \mathrm{Ga}) \mathrm{Se}_{2}$ solar cells. The Mo for this application has, in general, a high porosity [18], while Mo for other applications might require more dense layers and will react differently during laser texturing. The latter is not addressed in this work. 


\section{RESULTS}

\subsection{Experimental procedure}

The experimental procedure followed to determine the processing window for producing uniform areas covered by long and bifurcation-free LIPSS on thin Mo layers can be summarized in two subsequent steps. The first step was to find processing parameters to texture single laser tracks (lines) on the surface containing LIPSS. The second step was to find the optimal pitch (distance) between the single lines, required to generate uniform areas of bifurcations-free LIPSS.

During the first step, lines, scanned with different pulse OL, and number of OS at different fluence levels were produced. During these experiments the repetition frequency $f_{\mathrm{p}}$ was fixed at either 100 or $200 \mathrm{kHz}$ (Fig. 1). After initial SEM analysis to find LIPSS on damage-free layers, we iterated this step by choosing finer variation of the parameters.

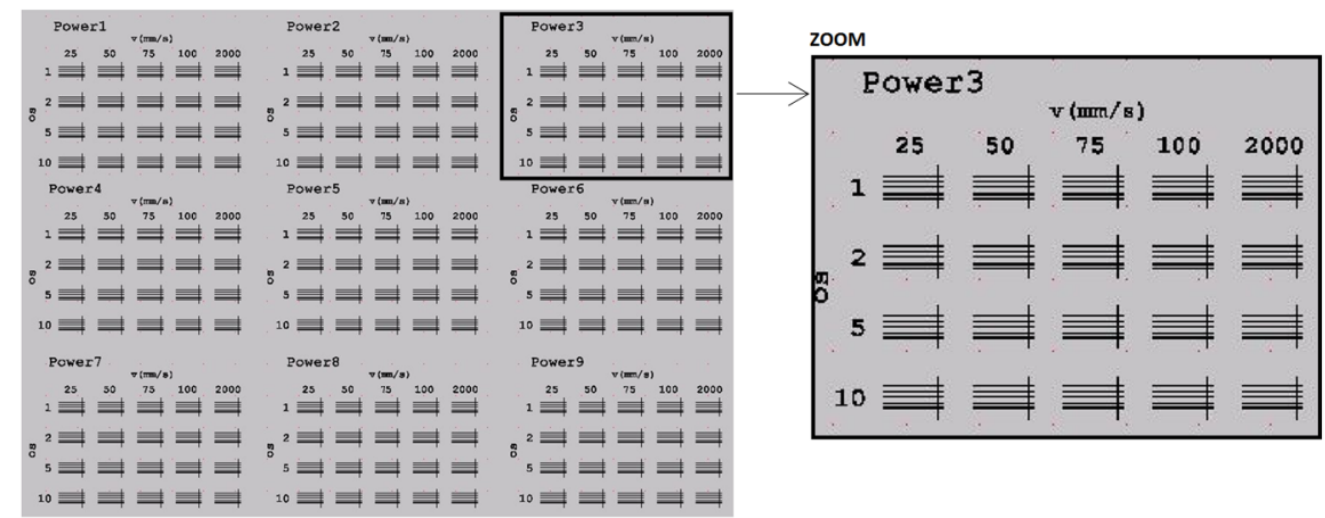

Figure 1. Matrix used to scan lines with different combinations of irradiation conditions. Repetition frequency was constant, while average laser power, speed and number of OS were varied. Pulse OL was calculated afterwards for different wavelengths using equation (1). The beam diameter, which was determined with the $\mathrm{D}^{2}$ method [4], was used as an input for equation (1). To check the reproducibility of the results, several identical lines were processed using identical conditions.

As mentioned in the introduction, the main goal was to reach the BDT in the layer, when trying to achieve damage free surface texturing of thin metal layers. This transition avoids cracks or peeling off (delamination) during, or directly after, laser processing. Whether BDT was reached, was found to be mainly determined by the pulse OL in the laser line as is illustrated in Fig. 2., where the fluence was kept constant and the increase of the OL -from none (single pulse) in Fig. 2(a) to $97,9 \%$ in Fig. 2(d)- significantly reduced the formation of cracks, delamination and chipping. It was found that high OL values $(>95 \%)$ allowed the deposited fluence $\left(\mathrm{J} / \mathrm{cm}^{2}\right)$ to be in the right range to create the LIPSS, without exceeding the single shot energy threshold to chip the thin layer.

After SEM analysis, single laser lines written at $200 \mathrm{kHz}$, instead of $100 \mathrm{kHz}$-while applying the same pulse energy, pulse duration, number of OS and OL- were found to be identical; therefore, we concluded that thermal accumulation effects between subsequent pulses, both at $100 \mathrm{kHz}$ and $200 \mathrm{kHz}$ played no role. In addition, we concluded that reaching the BDT state can be attributed to a reduced surface reflectivity of the surface after the first shots, resulting in increased absorbed energy; this results in the correct temperature range for BDT in the layer, which is needed to overcome thermal stresses during laser processing, otherwise the layer would be damaged.

To study the effect of pulse duration on obtained LIPSS, the pulse length was varied from $230 \mathrm{fs}$, through $1 \mathrm{ps}, 6.7 \mathrm{ps}$ and $10 \mathrm{ps}$. For $230 f s$ and $1 \mathrm{ps}$, no conditions were found resulting in LIPSS or they just occurred leaving the glass exposed in the valleys between peaks (Fig.3). At pulse durations of $6.7 \mathrm{ps}$ and $10 \mathrm{ps}$ the processing window for obtaining LIPSS was found to be relatively large. For these two pulse lengths, the produced textures were smoother and more superficial, i.e. the valleys were less deep and did not expose the glass substrate. Therefore, to create the large LIPSS areas, the used pulse length was $6.7 \mathrm{ps}$. The fact that these LIPSS are smoother and more superficial could probably be attributed to the relatively short electron-phonon coupling time $\tau$ of Mo compared to the pulse duration. The coupling 
time $\tau$, computable from data [6], is shorter than $1 p s$ for Mo for an electron temperature of $\mathrm{T}_{\mathrm{e}}<20000 \mathrm{~K}$, which we estimated to be the maximum temperature reached during the laser-texturing process. When the pulse duration is longer than $\tau$, the electronic conductivity allows heat diffusion to occur during the laser pulse, dissipating part of it and this leads to a shallower removal of Mo for pulse durations longer than $1 \mathrm{ps}$.

The second step of the procedure consisted of choosing a few sets of processing parameters from step one (section 3.1), and creating areas of LIPSS by controlling the pitch between parallel lines, scanned in the same direction. The OL between lines differed from the pulse OL in a single line, and here is referred to as the pitch. It is known that, LIPSS grow perpendicular to polarization of the laser light. Long LIPSS, without bifurcations and interruptions, can be obtained if the linear polarization of the electromagnetic field of the laser radiation is perpendicular to the scanning direction. In this way, LIPSS growth was not constrained by the width of the single laser line.
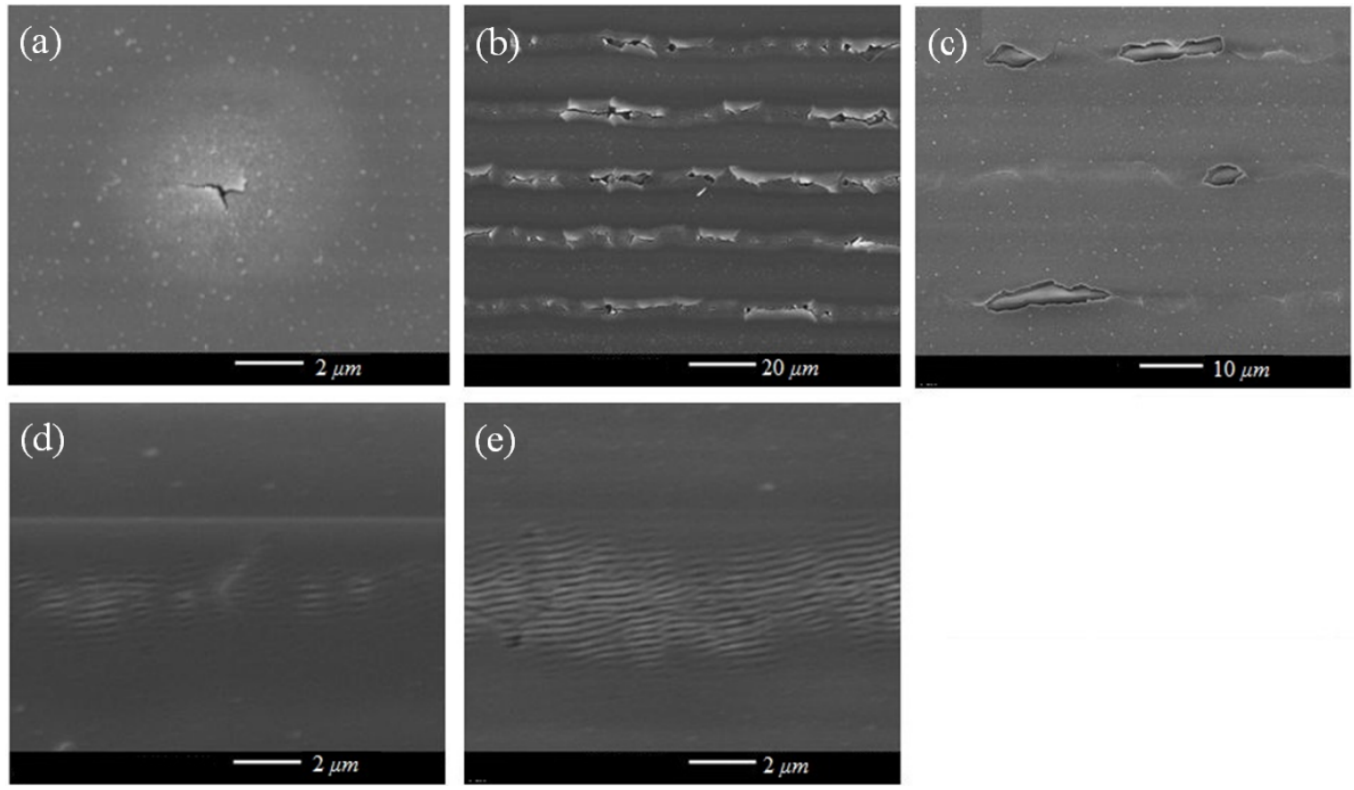

Figure 2. SEM images of $150 \mathrm{~nm}$ PVD deposited Mo layer on soda lime glass textured by $6.7 \mathrm{ps}$, $U V$ (343 $\mathrm{nm})$ laser pulses, where OS and scanning speed were varied at constant repetition frequency $(100 \mathrm{kHz})$ and constant fluence $0.05 \mathrm{~J} / \mathrm{cm}^{2}$. (a) $1 \mathrm{OS}$, single pulse (b) $1 \mathrm{OS}, 91.6 \% \mathrm{OL}$, (c) $1 \mathrm{OS}, 93.7 \% \mathrm{OL}$, (d) $1 \mathrm{OS}, 97.9 \% \mathrm{OL}$, (e) 2 OS, 97,9\% OL. Only at high pulse OL layer damage is avoided.
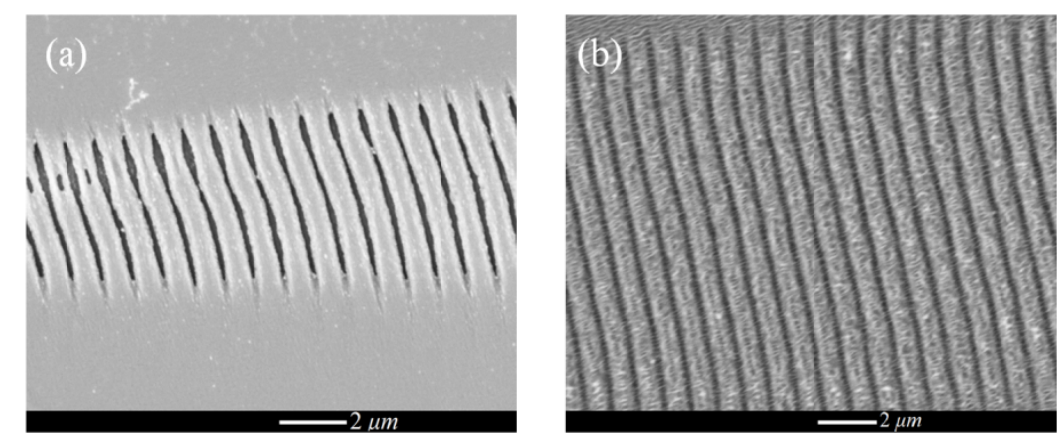

Figure 3. SEM pictures of LIPSS single lines on $400 \mathrm{~nm}$ Mo layer irradiated with IR light. (a) Pulse length $=0,23 \mathrm{ps}$, fluence $=0.81 \mathrm{~J} / \mathrm{cm}^{2}, 93 \%$ OL, 1 OS. (b) Pulse length $=10 \mathrm{ps}$, fluence $=1.26 \mathrm{~J} / \mathrm{cm}^{2}, 93 \%$ OL, 1 OS. LIPSS produced with $0.23 \mathrm{ps}$ are more steep and expose the glass substrate in the valleys, while LIPSS produced with 10 ps are smoother and more superficial. In our experiments, control over the pulse length of the laser's pulses enabled to avoid exposure of the glass substrate. Single arrow indicates the scanning direction, while double arrow indicates the polarization. 


\subsection{Obtained nanostructures and analysis}

Finally, conditions were found at which the surface was covered with long LIPSS without bifurcations, as shown in Fig. 4 (a). On the same sample, it was determined by CLSM that the material removed by ablation of the processed surface was below $150 \mathrm{~nm}$; the small quantity of removed material is a proof of the feasibility of laser texturing of thin Mo films in the used range of layer thicknesses. When required, the thinning of the processed layer could be compensated by using a thicker layer.

The obtained nanogratings were inspected by SEM and AFM to determine the periodicity and amplitude of the LIPSS obtained with UV, Green and IR laser light (Table 1). Their 3D morphology is shown in Fig. 4 (b); in Fig. 4 (c), the cross section of Fig. 4 (b) shows clearly their smooth sinusoidal shape. The feasibility of laser texturing of thin metallic films is demonstrated in Fig. 5 (a), where the uniform colourful effect of diffracted light displays the uniformity of LIPSS over a wide area. Fig. 5 (b) shows the AID of the gratings of Fig. 5 (a). The measured data from Fig. 5 (b) match with a nanograting of $390 \mathrm{~nm}$ periodicity, which is the same value observed with SEM and AFM inspection. Hence, this confirms that the optical diffraction shown in Fig. 5 (a) originates from the LIPSS and not from grooves formed by ablation during laser processing, which would show a longer periodicity.
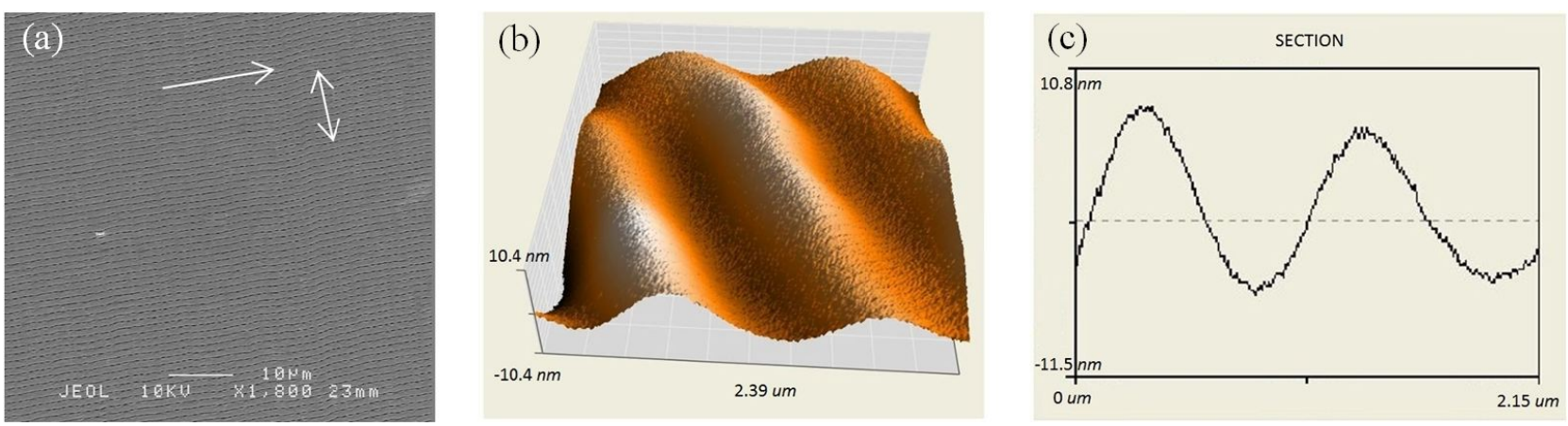

Figure 4. (a) SEM image of a $870 \mathrm{~nm}$ grating composed by highly periodical LIPSS on $400 \mathrm{~nm}$ Mo layer sputtered on soda lime glass. Processing conditions: $\lambda=1030 \mathrm{~nm}, 6.7 \mathrm{ps}, 98 \% \mathrm{OL}, 0.013 \mathrm{~J} / \mathrm{cm}^{2}, 100 \mathrm{kHz}, 0.3 \mu \mathrm{m}$ pitch between different lines and $30 \mu \mathrm{m}$ spot size. The length of the LIPSS is not limited by the laser spot's dimension. Single arrow indicates the scanning direction, while double arrow indicates the polarization. (b) AFM image showing the smooth 3D topography of the same sample. (c) 2-D cross-section of Fig. 4(b).
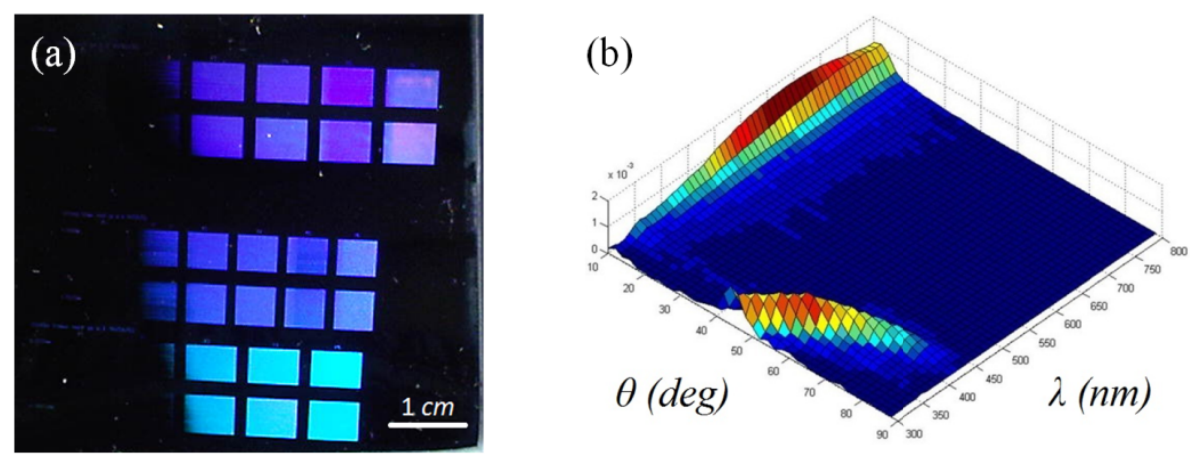

Figure 5. (a) Photograph of laser textured sample showing the feasibility of large area nanogratings (LIPSS) on $400 \mathrm{~nm}$ Mo layer. b) AID obtained by spectrophotometry as function of the scattering angle $\theta$ and the wavelength $\lambda$ of the incident light. Measured data match with a grating of $390 \mathrm{~nm}$ periodicity. 
Table 1. Averaged periodicity and amplitude of LIPSS obtained with the three different laser wavelengths. Data were obtained by AFM and SEM.

\begin{tabular}{|c|c|c|c|} 
& UV $[\lambda=343 \mathbf{n m}]$ & Green $[\lambda=\mathbf{5 1 5} \mathbf{n m}]$ & IR $[\boldsymbol{\lambda}=\mathbf{1 0 3 0} \mathbf{n m}]$ \\
\hline Averaged periodicity $[\mathrm{nm}]$ & 270 & 390 & 890 \\
\hline Averaged amplitude $[\mathrm{nm}]$ & 29 & 21 & 13 \\
\hline
\end{tabular}

\section{DISCUSSION}

Theoretical and numerical models of LIPSS, or more specifically models predicting periodicity of LIPSS are known only for bulk material. In this section, we compared the periodicity predicted by one of those "bulk" models to the experimentally obtained periodicity in the thin Mo layers.

In 1973, it was proposed by Emmony et al. [12] that LIPSS were a result of interference between the incident laser beam and surface-scattered waves. On the basis of this concept, Sipe et al. developed the efficacy factor theory in 1983 [13]. This theory is usually referred to as Sipe theory. It predicts, in the frequency domain, the spatial inhomogeneous energy absorption $A(\boldsymbol{k})$ just below the rough surface of materials as a function of $\boldsymbol{k}$. Here, $\boldsymbol{k}$ is a vector spanning the frequency domain, normalized by the norm of the wave vector of the incident laser light $2 \pi / \lambda$. The main assumption is that LIPSS occur where the absorbed energy $A(\boldsymbol{k})$ is the largest. In the frame of the Sipe theory, $A(\boldsymbol{k})$ is proportional to $\eta(\boldsymbol{k}) \times b(\boldsymbol{k})$, where $b(\boldsymbol{k})$ is the Fourier component of the initial roughness of the surface and $\eta(\boldsymbol{k})$ is the so-called Efficacy Factor. This factor quantifies the efficacy with which the roughness leads to an inhomogeneous absorption at $k$. The expression of $\eta(\boldsymbol{k})$ can be found in the original article of Sipe et al. [13]. Prior to any laser treatment, $b(\boldsymbol{k})$ is expected to be a slowly varying function for a surface with homogeneously distributed roughness [13]. Hence, the inhomogeneous absorption of energy from the laser radiation leading to LIPSS formation is mainly governed by the quantity $\eta(\boldsymbol{k})$.

An example of $\eta(\boldsymbol{k})$, as a function of frequency components in two directions ( $x$ and $y$ ) is shown in Fig. 6 (a). It was computed for the same parameters as in the experiments in section 3 . That is, $\lambda=515 \mathrm{~nm}$, angle of incidence $\theta=0$, optical materials properties $\tilde{\mathrm{n}}=3.192+3.378 i(\tilde{n}=n+i k)$, statistical surface roughness parameters $F=0.1$ and $s=0.4$. Here, $F$ is the filling factor and $s$ the shape factor. $F$ and $s$ are parameters used to describe random rough surfaces. More information can be found in [13] and [15]. The complex refractive index is that of Mo for $\lambda=515 \mathrm{~nm}$ [17]. To discuss the results in the frequency domain, the same notations as in [16] are used. The only visible features in Fig. 6 (a) are the so-called type-s features. These features are following the bright areas of the outer part of the circle of radius $\|\boldsymbol{k}\|=1$, meaning that the LIPSS expected on Mo under these laser conditions should have a periodicity slightly smaller than $\lambda=$ $515 \mathrm{~nm}$.
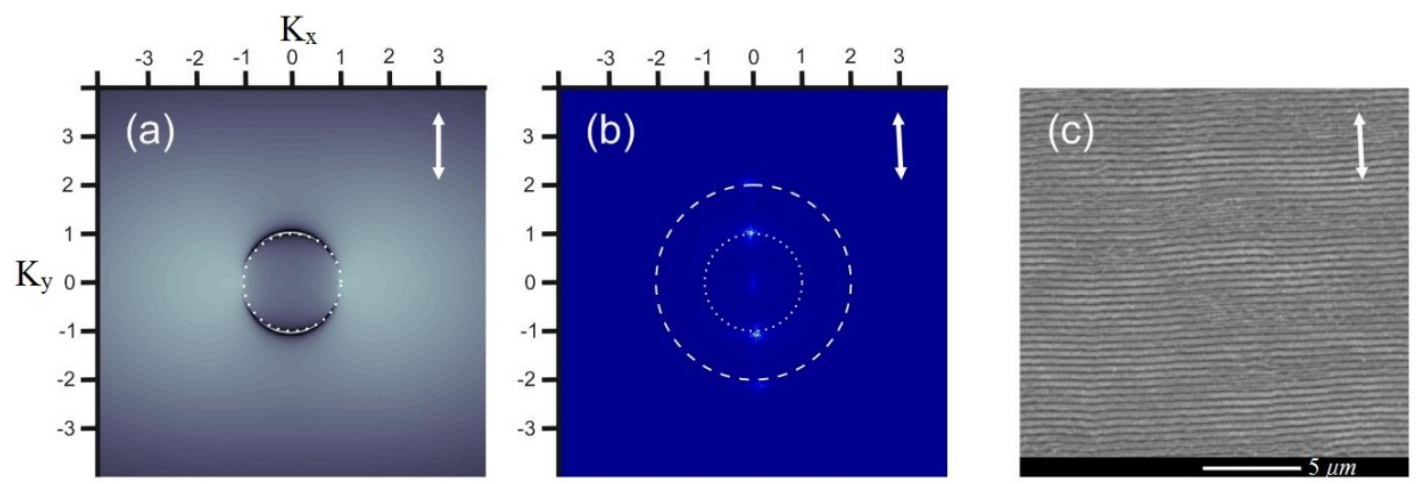

Figure 7. (a) Efficacy factor map computed with $\lambda=515 \mathrm{~nm}, \theta=0, n=3.192, k=3.378, F=0.1$ and $s=0.4$. A linear gray scale is used. (b) Fast Fourier transform of (c). (c) Large area of ripple obtained on $400 \mathrm{~nm}$ thick Mo layer with $0.009 \mathrm{~J} / \mathrm{cm}^{2}, f=200 \mathrm{kHz}, 98 \% \mathrm{OL}$ and $20 \mu \mathrm{m}$ pitch between scanned lines. The dotted circles in (a) and (b) have radii $k=1$. The dashed circle in b) has a radius $k=2$. The polarization direction is indicated by the white arrows in (a), (b) and (c). 
In Fig. 6 (c), LIPSS produced on $400 \mathrm{~nm}$ thick layer with $0.009 \mathrm{~J} / \mathrm{cm}^{2}, f=200 \mathrm{kHz}, 98 \%$ OL and $20 \mu \mathrm{m}$ pitch between scanned lines are shown. The 2D Fourier transform of the intensities in Fig. 6 (c) is presented in Fig. 6 (b). Next, this frequency map can be compared to the predicted $\eta(\boldsymbol{k})$-map in Fig. 6 (a). The type-s in Fig. 6 (b) are following the circle of radius $\|\boldsymbol{k}\|=1$ as in the efficacy factor map. However, they are less spread in the frequency domain. This is not in contradiction with the Sipe theory since the predictions in Sipe's theory were made for initially random rough surfaces. Once the LIPSS start to develop, $\eta(\boldsymbol{k})$ is not the only quantity driving LIPSS growth, because then $b(\boldsymbol{k})$ is no longer a slowly varying function. Other interesting features are visible in Fig.6 (b). Bright areas (so relevant frequencies in the absorbed energy) are following the outer part of the $\|\boldsymbol{k}\|=2$ circles. These frequencies have an amplitude significantly smaller than the type-s features. These correspond to LIPSS forking in the 2D spatial domain. In a recent article, Skolski et al. showed that the type-s are not the only features contributing to the periodicity to be expected in the space domain. These other features are referred to [16] as type-r. Even though, the simulations here were made for a material for which $n>k, \eta(\boldsymbol{k})$ is also showing type-r features for a material if $n<k$. Therefore, we attribute the features around the $\|\boldsymbol{k}\|=2$ circle to the type-r features. It is worth mentioning that a study of the interpulse feedback mechanisms involved in LIPSS formation would clarify these experimental observations. Nonetheless, we concluded from the comparison of the "bulky" modeling results and the experimental results, that LIPSS produced in the thin Mo layers have the same origin as the LIPSS formed on bulk materials.

\section{CONCLUSIONS}

We demonstrated the feasibility of LIPSS very regular nano gratings of various periodicities $(890 \mathrm{~nm}$ and $390 \mathrm{~nm})$ over large areas on $400 \mathrm{~nm}$ thin Mo film on borosilicate glass using $6.7 \mathrm{ps}$ laser without cracking the film nor exposing the glass.

We also showed that the periodicity of LIPSS produced on thin films was in good agreement with the theoretical expectations for LIPSS on bulk material.

A drawback of this technology is the low speed required to process large areas (e.g., using a $1 \mathrm{~kW} \mathrm{ps} \mathrm{laser,} \mathrm{texturing} 1$ $\mathrm{m}^{2}$ of surface requires about $50 \mathrm{~s}$ ). Although laser texturing is probably not a promising technology in PV industry due to the high area output required, thin metal film surface texturing can be an interesting solution for other applications such as nano-optics or microelectronics.

\section{AKNOWLEDGEMENTS}

This work was done within the Advanced Dutch Energy Materials Innovation Lab (ADEM) programme, funded by the Dutch Ministry of Economic Affairs, Agriculture and Innovation. The authors also want to thank ECN, J. Bosman, C. de Kok. The contributions of Frits de Lange and B. Pathiraj are acknowledged.

\section{REFERENCES}

[1] W. Soppe, F-J Haug, P. Couty, M. Duchamp, W. Schipper, J. Krc, G. Sanchez, K. Leitner, Q. Wang, $26^{\text {th }}$ Europ. Photovoltaic Solar Energy Conf. and Exhib., Hamburg, Germany (2011)

[2] M. Birnbaum, J. Appl. Phys. 36(11), 3688-3689 (1965)

[3] F. Garrelie, J. P. Colombier, F. Pigeon, S. Tonchev, N. Faure, M. Bounhalli, S. Reynaud, O. Parriaux, Optical Society of America, 19 No.10, (2011)

[4] J. Bonse, J.M. Wrobel, J. Krüger, W. Kautek, Appl. Phys. A, 72, 89-94, (2001)

[5] K J ger, O Isabella, R A C M M van Swaaij, M Zeman, Meas. Sci. Technol. 22 (2011)

[6] http://faculty.virginia.edu/CompMat/electron-phonon-coupling/

[7] J. Reif, F. Costache, M. Henyk, S. V. Pandelov, Appl. Surf. Sci. 197-198, 891 (2002)

[8] M. Huang, F. L. Zhao, Y. Cheng, N. Xu, Z. Xu, Phys. Rev. B 79, 125436 (2009)

[9] D. Dufft, A. Rosenfeld, S. K. Das, R. Grunwald, J. Bonse, J. Appl. Phys. 105, 034908 (2009)

[10] J. Bonse, A. Rosenfeld, J. Krueger, J. Appl. Phys. 106, 104910 (2009) 
[11] A. Borowiec, H. K. Haugen, Appl. Phys. Lett. 82, 4462 (2003)

[12]D. C. Emmony, R. P. Howson, L. J. Willis, Applied Physics Letters 23, 598 (1973)

[13] J. E. Sipe, J. F. Young, J. S. Preston, H. M. van Driel, Physical Review B 27, 1141 (1983)

[14] J. Bonse, M. Munz, H. Sturm, Journal of Applied Physics 97, 013538 (2005)

[15] J. F. Young, J. S. Preston, H. M. van Driel, J. E. Sipe, Physical Review B 27, 1155 (1983)

[16] J. Z. P. Skolski, G. R. B. E. Römer, J. V. Obona, V. Ocelik, A. J. Huis in 't Veld, J. Th. M. De Hosson, Physical Review B 85, 075320 (2012)

[17] The Landolt-Bornstein Database, http://www.springermaterials.com/navigation/

[18] J. H. Scofield, A. Duda, D. Albin, B.L. Ballard, P.K. Predecki, Thin Solid Films, 260, 26-31 (1995)

[19] M. Huang, F. Zhao, Y. Cheng, N. Xu, Z. Xu, ACS Nano, 3 (12), 4062-4070 (2009)

[20] S.K. Lee, K.K. Yoon, K.H. Whang, S.J. Na, Surface and Coatings Technology, 113, 63-74 (1999) 